\title{
Textured Enhanced Image De-noising using Fast Wavelet Transform
}

\author{
E. Sasikala \\ Gokula Krishna College of Engineering \\ Sullurupeta, India
}

\author{
T. Anitha \\ Gokula Krishna College of Engineering \\ Sullurupeta, India
}

\begin{abstract}
The digital imaging technologies possess very hasty development that uses Giga-pixels to store an image. Image de-noising algorithms plays a significant role in the restoration process. In an image the texture regions are homogeneous and are composed of local descriptor, a trade off exist between visual quality of image and the enhanced texture regions. In the existing paper the Gradient Histogram Preservation (GHP) method on the enhanced image regions have a limitation where it cannot be directly applied to nonadditive noise removal such as Multiplicative Poisson Noise (MPN) and Signal-Dependent Noise (SDN) to overcome the limitation Fast Wavelet Transform is used. In this work an image is first added with different types of noise like Additive White Gaussian Noise (AWGN), Salt and Pepper Noise, Poisson Noise, Signal Dependent noise and Flicker Noise, the noisy image is restored using filters, next the enhanced texture region of the image is chosen which is blurred or deformed and the fine details of the texture is obtained using Fast Wavelet Transform (FWT). The proposed work is analyzed in Frequency domain by considering various parameters like Peak Signal to Noise Ratio (PSNR), Correlation Factor (CF) and Standard Deviation (SD) and the quality of the enhanced region of the image is improved to the best level than the conventional noise removal algorithms.
\end{abstract}

Keywords: Image Textures; Fast Wavelet Transform; Peak Signal to Noise Ratio; Correlation Factor; Standard Deviation.

\section{INTRODUCTION}

Image Noise models can be classified into two groups. They are (i) Image Independent Noise Model and (ii) Image Dependent Noise Model. Image independent noise can be described by an additive noise model by the equation 1 .

$$
f(i, j)=s(i, j)+n(i, j)
$$

Where $f(i, j)$ the processed image or Noisy image is, $s(i, j)$ is the true image without addition of noise and $n(i, j)$ is the additive noise on the true image. Noise $n(i, j)$ is often zero mean and described by its variance $\sigma_{n}^{2}$. Signal to Noise Ratio (SNR) is defined as

$$
S N R=\frac{\sigma_{s}}{\sigma_{n}}=\sqrt{\frac{\sigma_{f}^{2}}{\sigma_{n}^{2}}-1}
$$

In the above equation $2, \sigma_{s}^{2}$ the variance of true image and $\sigma_{f}^{2}$ is the variance of processed image. In several cases, white additive noise is evenly distributed over the frequency domain. Where white noise is one of the random signal as shown in Figure 1, having equal intensities at different frequencies and it has a constant power spectral density.

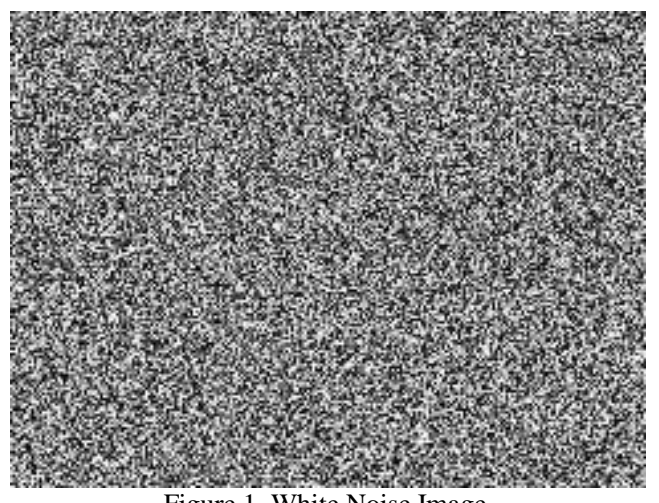

Figure 1. White Noise Image
Data or Image dependent noise model is done with a non linear model or multiplicative model. The image dependent noise models are more complicated, one of the example is an image resulted due to the addition of monochromatic radiation getting scattered from a surface whose irregularities or roughness is of the order of a wavelength which causes interference and it results in speckle noise.

A widespread signal-dependent noise model has been anticipated to deal with numerous diverse acquisition systems. Many types of noise can be described by using the following parametric model equation 3 .

$$
g(m, n)=f(m, n)+f(m, n) \gamma \cdot u(m, n)+w(m, n)
$$$$
=f(m, n)+v(m, n)+w(m, n)
$$

Where $(m, n)$ is the pixel location, $g(m, n)$ the observed noisy image, $f(m, n)$ the noise-free image, modelled as a nonstationary correlated random process, $u(m, n)$ a stationary, zero-mean uncorrelated random process independent of $f(m, n)$ with variance $\sigma 2 u$, and $w(m, n)$ is electronics noise (zero-mean white and Gaussian, with variance $\sigma 2$ ). For a great variety of images, this model has been proven to hold for values of the parameter $\gamma$ such that $|\gamma| \leq 1$. The additive term $v=$ $f \gamma \cdot u$ is the generalized signal-dependent (GSD) noise. Since ' $\mathrm{f}$ ' is generally non-stationary, the noise $\mathrm{v}$ will be nonstationary as well. The term $\mathrm{w}$ is the signal-independent noise component and is generally assumed to be Gaussian distributed. [2]

\section{PROBLEM STATEMENT}

The dilemma of getting better the regression function from noisy data based on wavelet decomposition. To restrain the noise in the data two approaches are normally used. The primary scheme is the called linear method. The wavelet decomposition echo's healthy the properties of the signal in the frequency domain. It is known that the higher decomposition scales related to higher frequency components in the regression function. . If we suppose that the fundamental regression is owed in the low frequency domain then the filtering process becomes evident. All empirical wavelet coefficients ahead of 
some resolution scale are anticipated by zero. This modus operandi works well if the signal is adequately smooth and whilst there is no edge effect in the data. But for several practical problems such a loom do not give the impression to be fully apposite, e.g. images cannot be painstaking as smooth functions.

To shun this inadequacy often a nonlinear filtering modus operandi is worn to hold back the noise in the empirical wavelet coefficients. The focal idea is based on the elementary property of the wavelet transform; father and mother functions are sound localized in time domain. Consequently one can estimate the empirical wavelet coefficients independently. To perform this approach compares the standard deviation of the noise and the absolute value of the empirical wavelet coefficient. It is lucid that if the wavelet coefficient is of the same order to that of the noise level, then it is difficult to separate the noise and the signal. In this state of affairs a high-quality estimator for the wavelet coefficient is nil. In the crate after an empirical wavelet coefficient is superior to the noise level a usual estimator for a wavelet coefficient is the empirical wavelet coefficient itself it is called thresholding. Hence divide the diverse adjustments of thresholding in principally three methods: Hard Thresholding, Soft Thresholding and a Levelwise Thresholding using Stein risk estimator.

\section{LITERATURE SURVEY}

The author proposed very effective denoising algorithm based on gradient histogram preservation which shows good PSNR/SSIM value. This approach is very logical working as a comparator circuit of OP-AMP and it work globally for any noisy image mathematically by using adaptive algorithm. This method is not applicable for multiplicative Poisson noise and signal dependent noise [1].

In this method various optical imaging instrument such as CCD (Charge Coupled Device) cameras, multispectral scanners and imaging spectrometers are used to estimate the noise. It depends on multivariate regression such as mean and variance. The method provides good accuracy, SNR and it is robust to the image textures which lead to over estimation of noise [2].

When the data samples are finite or quantized and nonparametric in nature then the noise estimation is done commonly using kernel density estimation where if the data samples are in parametric in nature then it is estimated empirically. Here Gradient Decent and expectation maximum techniques for max. Likelihood optimization is developed and it results in good smooth optimization [3].

In this method the digital image is degraded and restored using non linear filters called histogram adaptive fuzzy filter. The process is carefully checked and compared using other methods like Adaptive fuzzy mean filter, minimum-maximum detector based filter, minimum-maximum exclusive filter and weighted adaptive fuzzy mean. The PSNR is best compared with above methods [4].

Here the author concentrated on image and video signal for denoising the work is done using wavelet thresholding methods for removing various types of noises on the image and video signals. The various parameters like PSNR, correlation factor and MSE are calculated. [5]

\section{IMAGE DENOISING BLOCK DIAGRAM}

In the process of image noising the non linear noise model is used where the noise is added in the channel being used to propagate the image signal. Here uncompressed image of TIFF (Tagged Image File Format) is used for processing the main objective is to remove the noise present on the image which got attacked in the noisy channel. Fast wavelet transforms like haar, $\mathrm{db} 2, \mathrm{db} 4, \mathrm{db} 6, \mathrm{db} 8$, Symlet, Cofilet and bi-orthogonal are used to remove the noise from the noisy image. The entire process is shown in the below figure 2 .

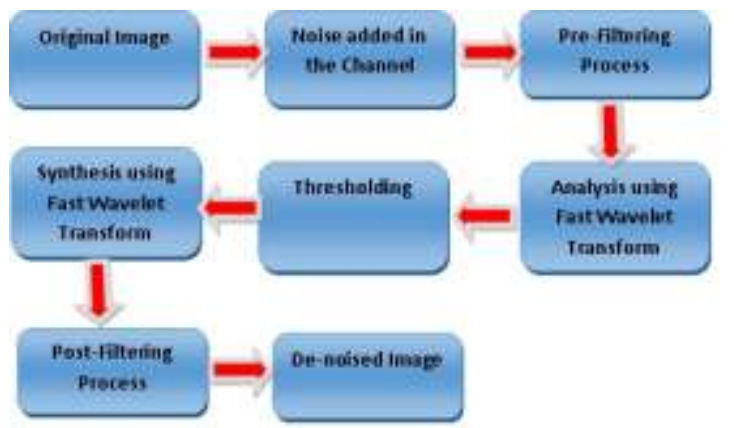

Figure 2. Image denoising block diagram

\subsection{Hard Thresholding}

To hold back the noise relates the following nonlinear transform to the empirical wavelet coefficients as shown in the equation 4 .

$$
F(x)=x \cdot I(|x|>t)
$$

Where ' $t$ ' is a firm threshold. The selection of the threshold is a very fragile and vital statistical problem. On one hand, a immense threshold guides to a huge bias of the estimator. Nevertheless on the other hand, a tiny threshold increases the variance of the smoother. Hypothetical concern capitulate the threshold as shown in equation 5 .

$$
t=\sqrt{2 \sigma^{2} \log (n) / n}
$$

Where ' $n$ ' is the length of the input vector and $\sigma^{2}$ is the variance of the noise

\subsection{Soft Thresholding}

The only difference between the hard and the soft thresholding measures are in the choice of the nonlinear transform on the empirical wavelet coefficients. For soft thresholding the following nonlinear transform is used

$$
S(x)=\operatorname{sign}(x)(|x|-t I(|x|>t))
$$

where ' $\mathrm{t}$ ' is a threshold. The set of choices provides you with all possibilities for choosing the threshold and discover the data.

\subsection{Adaptive Thresholding}

Adaptive thresholding using Stein's principle is as follows, We define a risk function $R\left(s_{t}, Z_{k}, t\right)$ with $Z_{k}=c_{k}+s_{k} e_{k}, k=$ $1,2, \ldots M$ where $c_{k}$ is the unknown coefficient, $s_{k}$ is the scaled parameter, $e_{k}$ is i.i.d $N(0,1)$ random variable and the threshold. Stein enables us to estimate the risk $\sum_{k=1}^{M} R\left(s_{k}, Z_{k}, t\right)$ the risk minimizing argument $\hat{t}$ can also be estimated and will be taken then as the optimal adaptive threshold ' $\mathrm{t}$ '.

$$
\hat{t}=\arg \min _{t \geq 0} \sum_{k=1}^{M}\left(2 s_{k}^{2}+t^{2}-Z_{k}^{2}\right) I\left\{\left|Z_{k}\right| \geq t\right\}
$$




\section{RESULTS}

The objective of image denoising is attained fruitfully with various thresholds. The below figure 3 shows the various noisy images like motion blurred image, blurred image,

Motion disk blurred image and Simulated blur and noise.
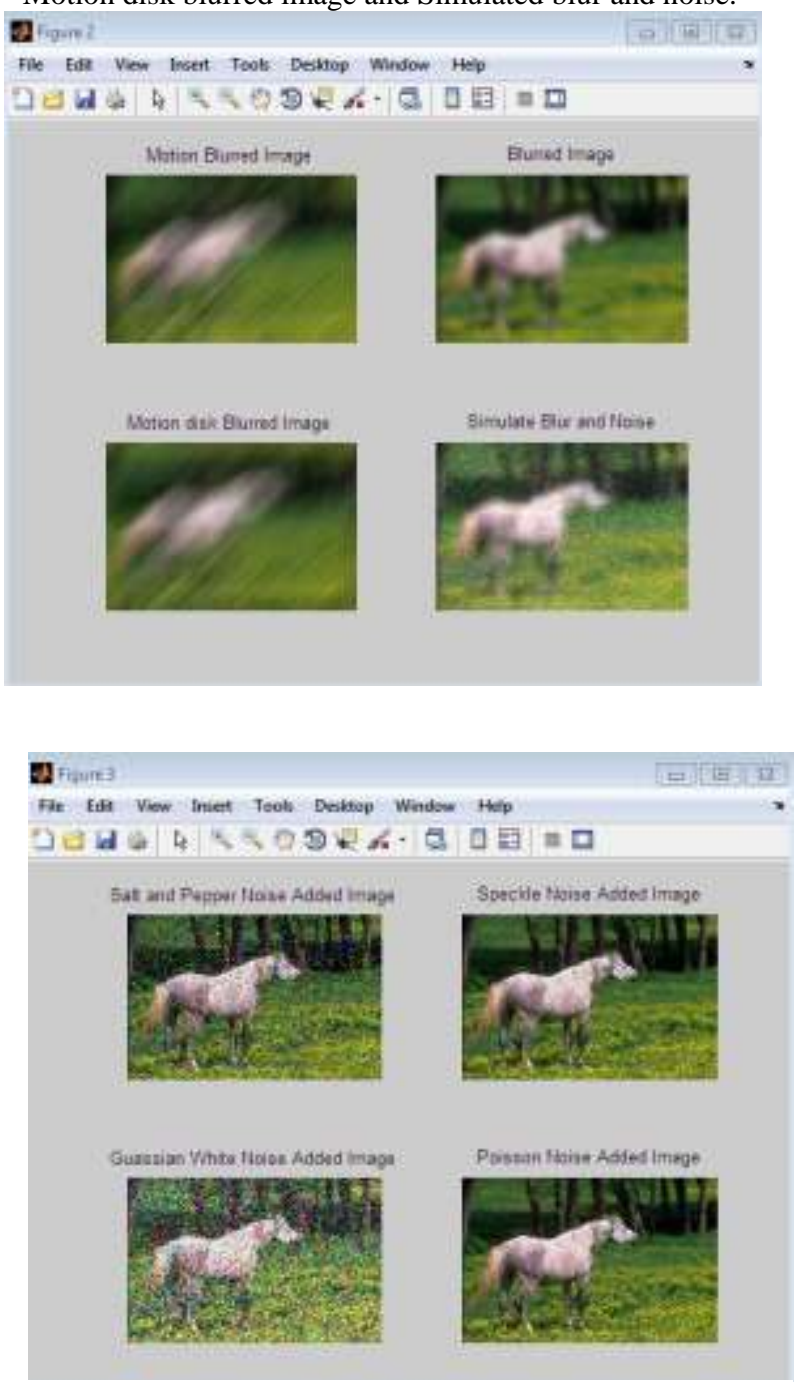

Figure 4. Different kinds of Noisy images

In the figure 4 salt and pepper noise, speckle noise, Gaussian white noise and Poisson Noise are added and respective figures are shown above. The PSNR of the results is shown in the below Figure 5.

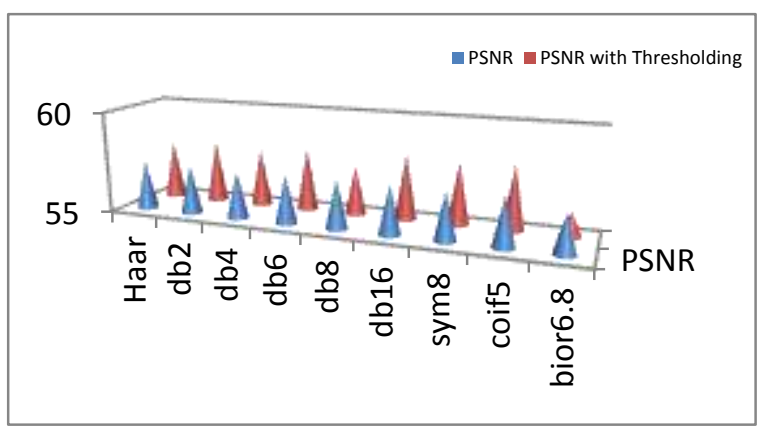

Figure 5. PSNR values without and with adaptive thresholding
Figure 3. Various Blur with Noises

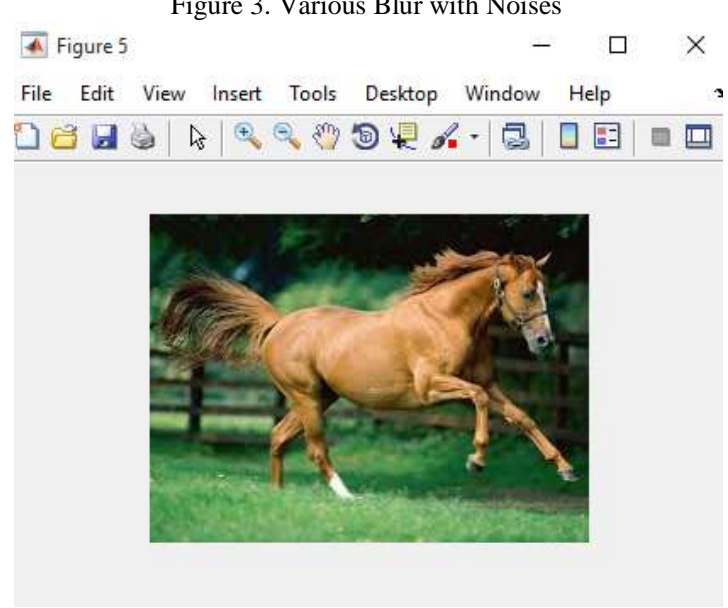

Figure 6 . Image processed by db16 wavelet.

The above figure 6 shows the image being processed with $\mathrm{db} 16$ wavelet for image denoising with adaptive thresholding method and figure 7 , shows the corresponding PSNR values in chart.

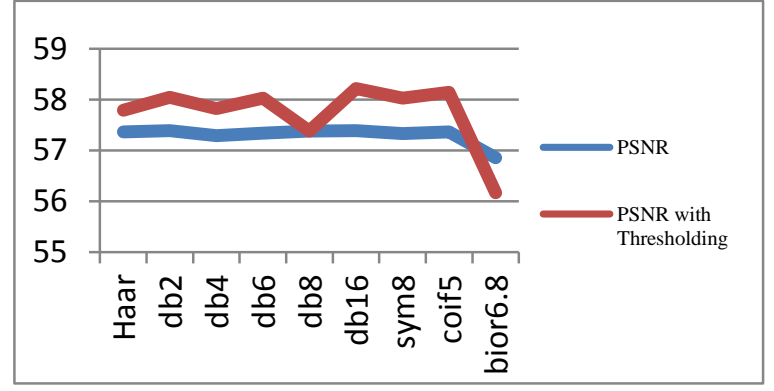

Figure 7. PSNR values of various wavelet transform

\section{CONCLUSION}

In the process of image denoising proper preprossing filter is used where the pre-processing filters are Median filer, Averaging filter, and mean filter. The best method to remove the signal dependent noise is to use wavelets in which the noise is removed in by using adaptive thresholding method. The PSNR value of different wavelets has been observed. The db8 and db16 wavelets are best suited for White Gaussian noise intensities, for speckle noise haar and family of daubechies wavelets can be used. The Best PSNR value is observed is 58.865 for Coiflet transform and daubechies transform with 58.85

\section{REFERENCES}

[1] Wangmeng Zuo, et. al., "Gradient Histogram Estimation and Preservation for Texture Enhanced Image Denoising" IEEE Transaction on Image Processing, vol. 23, No. 6, June 2014, pp. 2459 to 2472.

[2] Massimo Selva, et. al., "Signal-Dependent Noise Modelling and Estimation of Optical Imaging Instruments", University of Siena, Via Roma, 56, 53100 Siena, Italy.

[3] Nicol N. Schraudolph, "Gradient-Based Manipulation of Non-Parametric Entropy Estimates", IEEE transactions on neural networks, vol. 14 , no. 2, march 2004, pp. 1 to 10. 
International Journal of Computer Applications Technology and Research

Volume 5-Issue 11, 683-686, 2016, ISSN:-2319-8656

[4] Charu Khare, "Image Restoration Technique with Non Linear Filter", International Journal of Advanced Science and Technology, Vol. 39, February, 2012.

[5] Ayushi Jaiswal, et.al., "A Noval study on image and Video denoising" International Journal of Computer Application, Issue 4, Volume 3 (May-June 2014), ISSN: 2250-1797.

[6] R. Gonzales and R. Woods Digital Image Processing, Addison Wesley, 1992, pp 187 - 213.
[7] E. Davies Machine Vision: Theory, Algorithms and Practicalities, Academic Press, 1990, pp 29 - 30, 40 - 47. 493. 